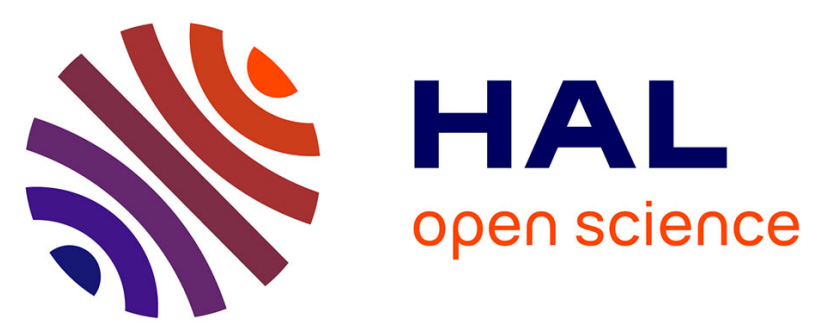

\title{
Advanced Modeling of Electromagnetic Loading of Cable-in-Conduit Conductors for Fusion Magnets
}

Rebecca Riccioli, Alexandre Torre, Damien Durville, Marco Breschi, Frédéric

Lebon, Vladimir Tronza

\section{- To cite this version:}

Rebecca Riccioli, Alexandre Torre, Damien Durville, Marco Breschi, Frédéric Lebon, et al.. Advanced Modeling of Electromagnetic Loading of Cable-in-Conduit Conductors for Fusion Magnets. IEEE Transactions on Applied Superconductivity, 2020, 30 (4), pp.1-5. 10.1109/TASC.2020.2978463 . hal02909285

\section{HAL Id: hal-02909285 \\ https://hal.science/hal-02909285}

Submitted on 8 Sep 2020

HAL is a multi-disciplinary open access archive for the deposit and dissemination of scientific research documents, whether they are published or not. The documents may come from teaching and research institutions in France or abroad, or from public or private research centers.
L'archive ouverte pluridisciplinaire HAL, est destinée au dépôt et à la diffusion de documents scientifiques de niveau recherche, publiés ou non, émanant des établissements d'enseignement et de recherche français ou étrangers, des laboratoires publics ou privés. 


\title{
Advanced Modeling of Electromagnetic Loading of Cable-in-Conduit Conductors for Fusion Magnets
}

\author{
Rebecca Riccioli ${ }^{(0)}$, Alexandre Torre ${ }^{(\mathbb{0})}$, Damien Durville, Marco Breschi ${ }^{\circledR}$, Frederic Lebon, and Vladimir Tronza
}

\begin{abstract}
The electrical performance degradation of $\mathrm{Nb}_{3} \mathrm{Sn}$ cables in the Cable-in-Conduit Conductors CICC has been well documented in literature. The $\mathrm{Nb}_{3} \mathrm{Sn}$ composite strands exhibit a critical current density that strongly depends on the strain state of the superconducting filaments. During a fusion magnet operation, the conductors are submitted to several electromagnetic and thermal cycles affecting the $\mathrm{Nb}_{3} \mathrm{Sn}$ mechanical state and consequently the capacity of the conductors to transport current. Different studies based on both a macroscopic and microscopic approaches have been performed so far to identify the mechanisms determining the conductors' behavior. Nevertheless, no theory permitting to predict the electrical performance of cyclically loaded conductors has been developed yet. Therefore, a solid electromechanical model able to tackle the analysis of CICC for fusion cables when they undergo thousands of cyclic loadings would be very useful. In this paper an advanced mechanical model to study the mechanical behavior of ITER TF CICC based on an improved version of the MULTIFIL finite element code is presented. A correction is introduced to solve the problem of the large impact of the boundary conditions in the simulation of the thermal loading, encountered in a previous work. A novel methodology to identify the value of thermal strain to be applied in cool-down simulations has also been developed. The model was adapted to take into account the Lorentz force cumulative effect of the other petals on the one under analysis. An assessment of the electromagnetic behavior based on the mechanical analysis is also presented.
\end{abstract}

Index Terms-CICC, $\mathrm{Nb}_{3} \mathrm{Sn}$, fusion, superconducting magnet.

Rebecca Riccioli is with the CEA, Commissariat à l'énergie atomique et aux énergies alternatives, 13108 Saint-Paul-Lez-Durance, France, and also with the Aix-Marseille University, 13007 Marseille, France, and also with the Bologna University 40126, Bologna, Italy (e-mail: rebecca.riccioli@cea.fr).

Alexandre Torre is with the CEA, Commissariat à l'énergie atomique et aux énergies alternatives, 13108 Saint-Paul-Lez-Durance, France (e-mail: alexandre.torre@cea.fr).

Damien Durville is with the Mechanics of Soils, Structures and Materials (MSSMat) Laboratory Centrale Supélec, CNRS UMR8579 Université Paris Saclay, 91190 Saint-Aubin, France (e-mail: damien.durville@ centralesupelec.fr).

Marco Breschi is with the Department of Electrical, Electronic and Information Engineering, University of Bologna, 40136 Bologna, Italy (e-mail: marco.breschi@unibo.it).

Frederic Lebon is with the CNRS, Centrale Marseille LMA (Laboratoire de Mécanique et Acoustique)Aix-Marseille Université, 13007 Marseille, France (e-mail: flebon@1ma.cnrs-mrs.fr).

Vladimir Tronza is with the IO (ITER Organization), 13108 Saint-Paul-lezDurance, France (e-mail: vladimir.tronza@iter.org).

Color versions of one or more of the figures in this article are available online at http://ieeexplore.ieee.org.

\section{INTRODUCTION}

$\mathbf{T}$ OKAMAK reactors enable fusion reactions, very hot temperatures must be achieved in the plasma that has to be confined through intense magnetic fields. Very high current conductors $(\sim 68 \mathrm{kA})$ are needed, imposing magnets made with superconductors to avoid huge electrical power dissipation. The critical current density of $\mathrm{Nb}_{3} \mathrm{Sn}$ based superconducting wires is not only a function of temperature and magnetic field, but also of the superconductor strain state [1]. Even though the critical current density variation with magnetic field and temperature is well known for a superconducting wire [2], it is much more complex for a cable made of thousands of wires. In particular, the cable performance is tightly linked to the individual strands strain state, and to its evolution with operating loads [3].

As of today, there is no method or tool, except full scale test campaigns, to foresee the impact of the mechanical state on the critical current density of the cable due to the mechanical loadings. This paper is the continuation of a previous work [4] having as a main goal to create an electromechanical model to simulate the behavior of a Cable-In-Conduit Conductor (CICC) in operation. The model presented here was implemented in an upgraded version of the MULTIFIL code [5], and corrects the creation of artificially high local compressive state at one end of the cable during the cool-down simulations [4]. This work also addresses two other modeling issues. On one hand, a study was performed to determine the value of thermal strain to be adopted in simulations of the conductor cool-down with MULTIFIL. On the other hand, since MULTIFIL so far describes only one sub-cable of the CICC (the so called 'petal', belonging to the last cabling stage), a novel methodology was developed to account in simulations for the cumulated Lorentz force effect of the other sub-cables on the one under analysis. To this purpose, two different analytical models were used to identify the input boundary conditions for electromagnetic (EM) cycle simulations with MULTIFIL.

\section{MODEL HYPOTHESES}

\section{A. ITER TF Cable-in-Conduit Conductor}

Simulations performed in this paper are based on a geometry following the design of the CICC for the TF coils of the ITER project. These cables consist of 1422 strands twisted in a multi-stage configuration, with different twist pitches for each stage. The wires are enclosed into a stainless steel jacket that is compacted until a void fraction around $30 \%$ is reached. The cable can be divided into six main sectors of 237 strands, called 'petals', which represent the last cabling stage. Each petal is composed of 150 superconducting $\mathrm{Nb}_{3} \mathrm{Sn}$ composite strands and 87 copper strands. The six petals are twisted around a central 
steel spiral. The strand diameter is $0.82 \mathrm{~mm}$, the inner jacket radius $19.6 \mathrm{~mm}$ and the outer spiral radius $5 \mathrm{~mm}$.

\section{B. MULTIFIL Simulations and Hypotheses}

Thanks to MULTIFIL, it is possible to study the mechanical strain state over the strands for the main phases of the conductor life. This includes cable manufacture, compaction inside the jacket, heat treatment at $650{ }^{\circ} \mathrm{C}$ to create the superconducting $\mathrm{Nb}_{3}$ Sn phase, thermal loadings due to cool-down to $4.5 \mathrm{~K}$ and the application of electromagnetic loadings. Here, the EM loading is relevant to the SULTAN test conditions (nominal current $68 \mathrm{kA}$, background field $11.78 \mathrm{~T}$ ) [6]. More details about how these phases are simulated are given in the previous work [4].

The model created in MULTIFIL takes into account just one petal of the cable to reduce the numerical computation time. The simulated petal is untwisted and has a reduced length of $300 \mathrm{~mm}$ comparable to the petal twist pitch in the conductor. The material constitutive laws for $\mathrm{Cu}$ and $\mathrm{Nb}_{3} \mathrm{Sn}$ strands (elastic for manufacturing and elastoplastic for the other phases) are taken at room temperature. The heat treatment is simulated by resetting the stress [7] and strain maps triggered by the cabling and compaction stage. The cool-down $\left(600{ }^{\circ} \mathrm{C}-4.5 \mathrm{~K}\right)$ is simulated by applying to the petal an axial compression through the boundary conditions. Up to now, the Lorentz force was simulated by applying uniform loads over the strands length acting orthogonally to the strands axis (strands trajectories are taken into account).

\section{EleCtromechanical Model UpgRades}

\section{A. Improvements in the Cool-Down Simulations}

The simulation of the cool-down stage is aimed to reproduce the global axial compression resulting from the differential thermal expansion between the jacket and the cable. To do this, an incremental axial displacement is applied to one end of the petal, while this is constrained inside a set of analytical surfaces, and the other end is fixed. These surfaces act as rigid obstacles, and, interacting with the strands through frictional contact, shape the assembly of wires into the desired 'petal' geometry. It was found out that the aforementioned problem encountered in a previous version of MULTIFIL (see [4]) was due to assuming as non-deformable the surfaces defining the obstacles around the petal. Under this assumption, the relative displacements between strands and the obstacles during compression is greater at the petal end subject to the applied displacement than at the opposite end, which in turn results in a decreasing average value of the axial load along the petal (see [4]). To overcome this artefact, it was shown in [4] that eliminating the friction between strands and the rigid surfaces allows one getting a more homogenous axial load distribution. This result is however not entirely satisfactory, given the presence of buckling phenomena due to the absence of friction.

In this work, a correction was applied to the calculation of relative displacements between the nodes on the strands and their corresponding contact points on the obstacles, assuming these last ones are not fixed, but can move in the longitudinal direction undergoing the same axial deformation prescribed to the petal. Frictional effects between the strands and the obstacles are then fully taken into account in the cool-down simulations, thus preventing local buckling. A more realistic strain distribution after cool-down is now computed by the code, as shown in Fig. 1

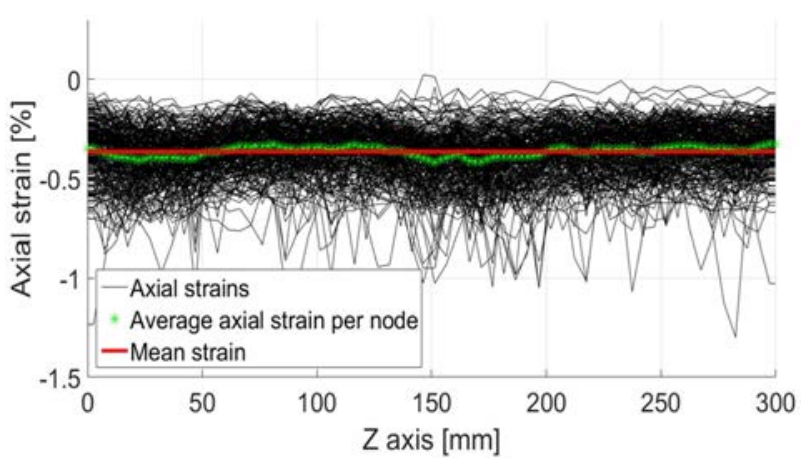

Fig. 1. Axial strain along the length of each strand (black) and its average value over all the strands (red) for an applied axial compression of $-0.84 \%$.

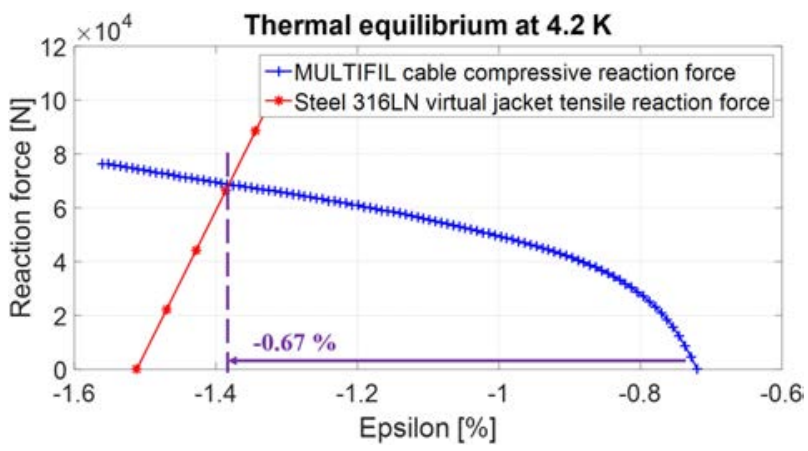

Fig. 2. Study of the thermal equilibrium at $4.2 \mathrm{~K}$ between the virtual stainless steel jacket in red and the simulated cable in blue. The equilibrium is reached at an applied strain to the cable of $-0.67 \%$.

for a global axial compression of $-0.84 \%$ applied to the cable. Thanks to this improvement, it is now possible to use MULTIFIL for the study of the longitudinal and the transversal reaction forces of the cable for a given applied load, since the frictional interactions between strands and obstacles do not affect the cool-down stage.

\section{B. Thermal Equilibrium Study}

The thermal strain is an input parameter for the cool-down simulation. A study to determine this value by computing the longitudinal reaction forces of the petal to an imposed compressive strain was performed. The methodology is based on finding the equilibrium, at a given applied strain, between these reaction forces (computed with MULTIFIL), and those arising in a virtual stainless steel jacket characterized by known values of Young's modulus (200.4 GPa, from [7]) and cross sectional area $\left(2.63 \cdot 10^{-4} \mathrm{~m}^{2}\right)$. Fig. 2 shows the tensile reaction force of the jacket computed analytically and the compressive reaction force found numerically. The starting points of the two curves correspond to the thermal properties at $4.2 \mathrm{~K}$ (also taken from [7]) of the stainless steel and $\mathrm{Nb}_{3} \mathrm{Sn}$. The starting thermal contraction mismatch at $4.2 \mathrm{~K}$ between the two materials is $-0.79 \%$; a value of strain equal to $-0.67 \%$ is identified to achieve mechanical equilibrium at the crossing point of the two curves.

\section{Multi-Petal Boundary Conditions}

One of the limitations of the model is the simulation of only one petal. When the electromagnetic loading is applied, it is 


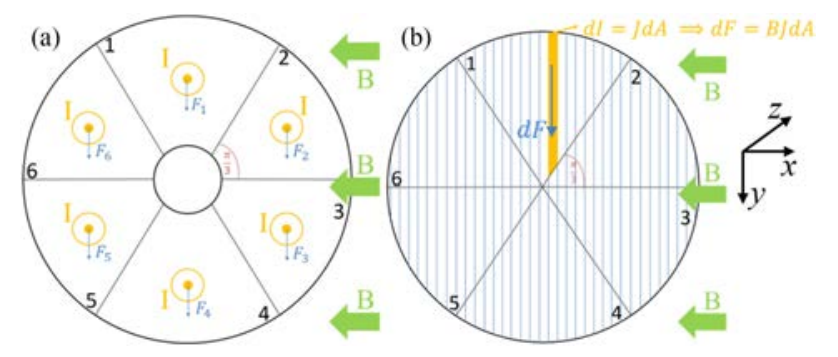

Fig. 3. (a) Full cable rigid analytical model for the EM loading and (b) full cable fluid analytical model for EM loading.

important to consider the cumulative effect of the Lorentz force of the other petals on the simulated one. To simulate this effect, we developed 'multi-petal' analytical models. The aim of these models is to identify the appropriate boundary conditions to be applied during the EM cycle simulations of one petal properly accounting for the loads applied by the other petals on the analyzed one. Two models have been developed, namely the rigid model (RM) and the fluid model (FM).

In the RM, the cable is composed of six petals interacting with each other like rigid bodies. The Lorentz force of each petal is applied at the petal center and the cumulated Lorentz force is transmitted to the other petals at the petals' interfaces. In the FM instead, the cable is described as a single entity, and is discretized into various columnar elements, each subjected to a specific EM force $d F$. The Lorenz force is unidirectional and acts similarly to a hydrostatic pressure; only the contribution to the Lorentz force of the elements located upon the studied petal is taken into account. In both models the petals are untwisted, the geometry is ideal, and the magnetic field is assumed as uniformly distributed. Fig. 3 illustrates the two models.

These two models do not give exactly the same results, since the force distributions are slightly different. To choose which one is more relevant to a given cable, it is reasonable to assume that the rigid bodies of the RM better represent the behavior of a cable with highly compacted and stiff petals, characterized by a strong mechanical bonding between strands. The FM seems instead more relevant to study a loose cable with less compaction. In general, the two models represent ideal limit cases; the behavior of a real cable should be included between these limits.

\section{Implementation of Boundary Conditions in MULTIFIL}

The models have been implemented in MULTIFIL for the study of the petal numbered as 4 in Fig. 3. To implement them, the idea is to move the flat surfaces of the petal in MULTIFIL until the stresses acting on them reach the values prescribed by the analytical models, thus corresponding to the accumulated load due to the other petals. The Fig. 4 shows the main direction of the surface displacements for both models.

Each petal is subjected to a Lorentz force of $133.5 \mathrm{~N} / \mathrm{mm}$; the analytical models prescribe the application on the flat surface of each petal of $400 \mathrm{~N} / \mathrm{mm}$ and $177 \mathrm{~N} / \mathrm{mm}$, for the RM and the FM respectively.

To reach these stresses on the surfaces, it was found that the surfaces have to displace from their starting position by $0.251 \mathrm{~mm}$ for the RM and $0.389 \mathrm{~mm}$ for the FM. The direction of the displacement changes depending on the model: in the RM the force is transmitted from petal to petal along the direction orthogonal to their separation surface, while in the FM the force
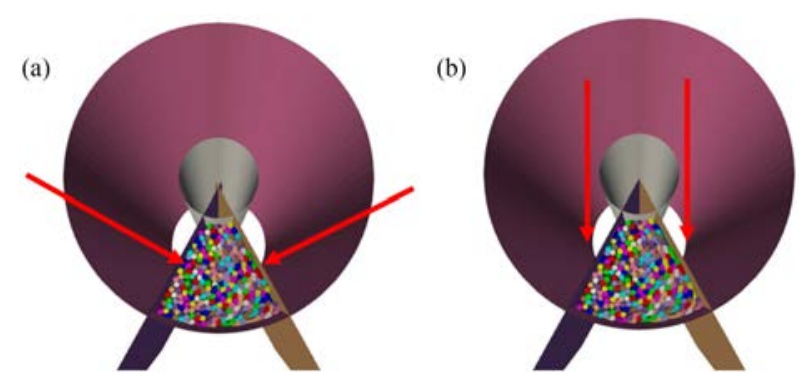

Fig. 4. (a) Implementation of the RM in MULTIFIL and (b) Implementation of the FM in MULTIFIL.

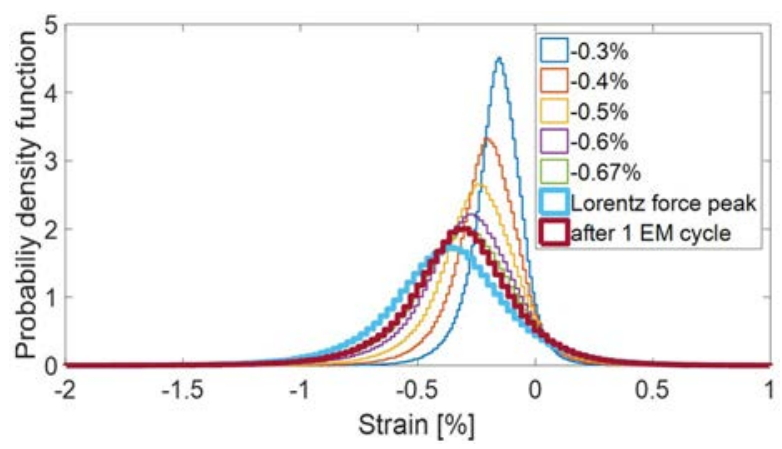

Fig. 5. Evolution of the probability density function during the compressive proceeding and the EM cycle $(68 \mathrm{kA}, 11.78 \mathrm{~T})$ for the RM test case.

is transmitted along the same direction as the Lorentz force (see Fig. 4).

To simulate the unloading phase of the EM cycle, the planes are moved back for both models.

\section{RESULTS}

The study of the simulation of one thermal loading due to the $650{ }^{\circ} \mathrm{C}-4.2 \mathrm{~K}$ cool-down and of one EM cycle with the $\mathrm{RM}$ at $(11.78 \mathrm{~T}, 68 \mathrm{kA})$ is reported here. For the cool-down a global axial compression of $-0.67 \%$ is considered, as found in the Section III-B. For the electrical analyses, the ITER scaling law [8] was adopted with the parameters corresponding to the SULTAN right leg for the TFIO1 tests campaign. For the strands an n-value of 25 was considered.

\section{A. Mechanical Results Analysis}

At the end of the cool-down computation an average axial strain of $-0.308 \%$ and an average bending strain of $0.307 \%$ were found. At the peak of the EM cycle the average axial strain is $-0.371 \%$ and the bending value is $0.368 \%$, while after the EM cycle they are respectively $-0.319 \%$ and $0.326 \%$.

It is possible to study the evolution of the strain distribution in the petal's strands during the different compressive steps and the EM cycle. Fig. 5 shows the gradual decreasing of the average strain during the cool-down and the parallel increasing of the distribution width. It seems that the EM cycle release allows recovering the average strain value of the last compressive step.

\section{B. Statistical Filaments Surface Study}

It is useful to analyze the $\mathrm{Nb}_{3} \mathrm{Sn}$ filamentary area in strands that could fracture in some computed strain ranges on the strands 

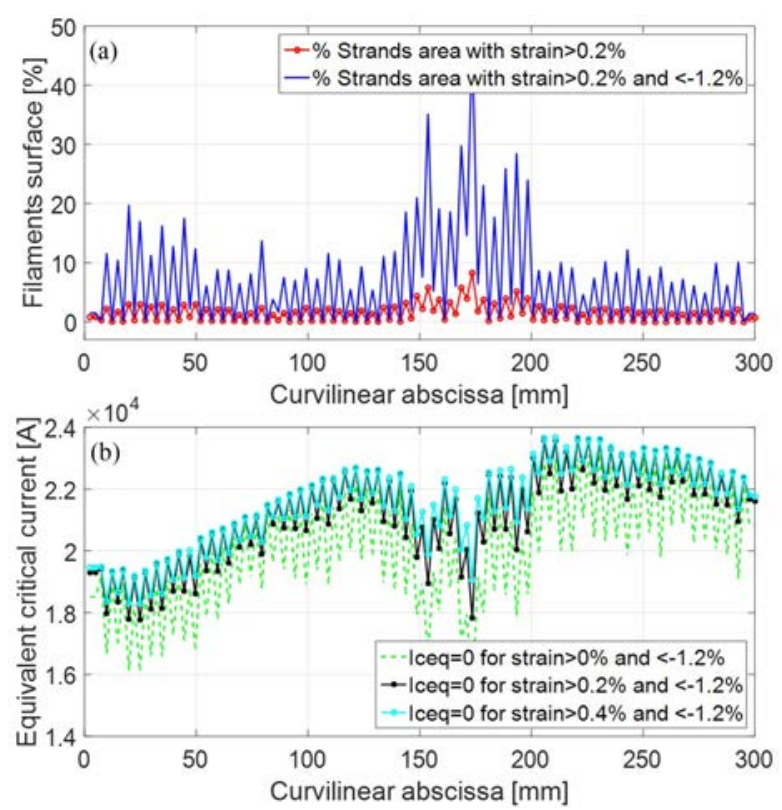

Fig. 6. (a) Percentage of filamentary surface after the EM cycle peak and (b) evolution of the equivalent critical current on the petal's cross-sections.

and the related variation of the critical current. In Fig. 6a), the percentage of filament surface per petal cross-section is reported after the peak of one EM cycle for two different strain ranges. Fig. 6b) shows the equivalent critical current per petal section for three different strain ranges considering a $T_{c s}$ of $6.5 \mathrm{~K}$ and a low resistivity limit analytical model for the current redistribution among the strands [9], [10].

\section{HRL and LRL Analytical Models for TCS Assessment}

A preliminary assessment of the $T_{c s}$ of the conductor as a function of different Lorentz force levels, can be performed and compared to those found in the SULTAN test campaign on the TFIO1 sample.

In order to find the $T_{c s}$ value, two electromagnetic analytical models are used to define the redistribution of the injected current inside the petal as a function of the simulated strain distribution. The two models represent two limiting cases: the Low Resistivity Limit (LRL) model (current can completely redistribute among strands) and the High Resistivity Limit (HRL) model (current cannot redistribute between strands) [9], [10].

The $T_{c s}$ in the LRL model is found by imposing the critical electric field $E_{c}$ on the whole petal and assuming that for each petal cross-section the critical current is given by the sum of the strands critical current. The main goal is to find the value of $T_{c s}$ corresponding to a current distribution which gives rise to an average $E_{c}=10^{-5} \mathrm{~V} / \mathrm{m}$ on the petal having a length $L$ :

$$
\begin{aligned}
& \langle E\rangle_{\text {petal_LRL }}=\frac{1}{L} \int_{z} E_{c} \\
& \quad \times\left(\frac{I_{p}}{\sum_{i}^{\text {strands on section }} I_{c i}\left(B, T_{c s L R L}, \varepsilon\right)}\right)^{n} d z=E_{c}
\end{aligned}
$$

In the HRL model, the $T_{c S}$ is found by imposing the critical electric field $E_{c}$ to each strand, so that a global critical current is defined for each strand. The purpose is then to find the $T_{c s}$

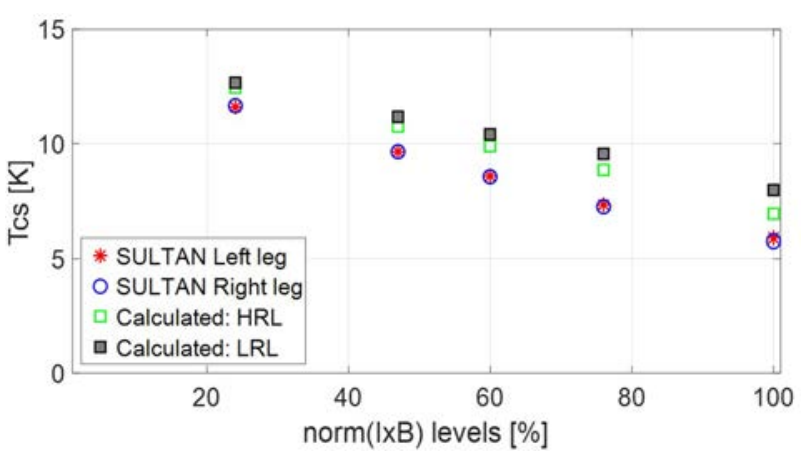

Fig. 7. Evolution of the $T_{c S}$ as a function of the Lorentz force level and comparison between the experimental SULTAN tests on the TFIO1 sample and the simulated results.

value corresponding to the $E_{c}$ on each strand, and therefore the current distribution through the sum over all the strands equal to the total current injected in the petal:

$$
I_{\text {petal_HRL }}=\sum_{i}^{\text {strands number }}\left(\sqrt[n]{\frac{L}{\int_{z}\left(\frac{1}{I_{c}\left(B, T_{c s H R L}, \varepsilon\right)}\right)^{n} d z}}\right)_{i}
$$

Fig. 7 shows a comparison between the simulated results and the SULTAN experimental ones for different Lorentz force levels. The simulations associated a critical current equal to zero to strain values above $0.2 \%$ and below $-1.2 \%$. The experimental tests included several EM cycles at each Lorentz force level. In this work only the first value of $T_{c s}$ found at each $I x B$ level has been compared to the simulation results.

As expected, the $T_{c s L R L}$ values are greater than the $T_{c s H R L}$ ones, since the current cannot redistribute in the HRL model if the strain in a strand entails a high local resistance. The trend of the computed results is in qualitative accordance with experimental results. The simulations presented here make use of a thermal strain value obtained through computation (differently from the fitting value adopted, for instance, in [11]).

\section{CONCLUSION}

Thanks to the upgrade of the thermal loading simulations with the MULTIFIL code, it is now possible to compute the thermal strain for the cool-down simulations, since the strain distributions are not affected by simulation artefacts. This upgrade of MULTIFIL gives access to the analysis of the mechanical properties of the cable, such as its transverse and longitudinal rigidities, which constitute a crucial property of the CICCs and will be studied in depth in future works.

For the first time also, the 'multipetal' analytical models allow to take into account in MULTIFIL the cumulated effect of the Lorentz force of the around petals on the one under study.

Thanks to the improvement of the electromechanical model, more reliable studies can be performed on the critical current evolution in each petal as a function of the percentage of filamentary surface at which the strain reaches extremal values, and on the $T_{c s}$ of the conductor. Two analytical electromagnetic models have been developed to investigate the evolution of $T_{c s}$ at different levels of the applied Lorentz force, as already done in the SULTAN test campaign of the TFIO1 sample, obtaining a good qualitative agreement with the measured trends. 


\section{REFERENCES}

[1] J. Ekin, "Effect of transverse compressive stress on the critical current and upper critical field of $\mathrm{Nb}_{3} \mathrm{Sn}$," J. Appl. Phys., vol. 62, no. 12, pp. 4829-4834, 1988.

[2] A. Nijhuis et al., "The effect of axial and transverse loading on the transport properites of ITER $\mathrm{Nb}_{3}$ Sn strands," Supercond. Sci. Technol., vol. 26, no. 8, 2013, Art. no. 084004.

[3] D. Ciazynski, "Review of $\mathrm{Nb}_{3}$ Sn conductors for ITER," Fusion Eng. Des., vol. 82, no. 5-14, pp. 488-497, 2007.

[4] R. Riccioli et al., "Mechanical modeling and first case study on ITER TF CICC loading cases with upgraded finite element code simulations," IEEE Trans. Appl. Supercond., vol. 29, no. 5, Aug. 2019, Art. no. 0603905.

[5] D. Durville, "Numerical simulation of entangled materials mechanical properties," J. Mater. Sci., vol. 40, no. 22, pp. 5941-5948, 2005.

[6] P. Bruzzone et al., "Status report of the SULTAN test facility," IEEE Trans. Appl. Supercond., vol. 20, no. 3, pp. 455-457, Jun. 2010
[7] N. Mitchell, "Finite element simulations of elasto-plastic processes in $\mathrm{Nb}_{3}$ Sn strands," Cryogenics, vol. 45, no. 7, pp. 501-515, 2005.

[8] Y. Ilyin et al., "Scaling law for the strain dependence of the critical current in an advanced ITER $\mathrm{Nb}_{3} \mathrm{Sn}$ strand," Supercond. Sci. Technol., vol. 20, no. 3, pp. 186-191, Jan. 2007.

[9] D. Ciazynski et al., "Analytical formulae for computing the critical current of an $\mathrm{Nb}_{3}$ Sn strand under bending," Supercond. Sci. Technol., vol. 23, no. 12, 2010, Art. no. 125005.

[10] J. Ekin, "Strain scaling law and the prediction of uniaxial and bending strain effects in multifilamentary superconductors," in M. Suenaga and A. F. Clark Eds., Filamentary A15 Superconductors, Cryogenic Materials Series, Boston, MA: Springer, 1980.

[11] M. Breschi et al., "Modeling of the electro-mechanical behavior of ITER $\mathrm{Nb}_{3} \mathrm{Sn}$ cable in consuit conductors," Supercond. Sci. Technol., vol. 25, no. 5, 2012, Art. no. 054005 . 